\title{
Superoleophobic Slippery Lubricant-Infused Surfaces: Combining Two Extremes in the Same Surface
}

\author{
Zheqin Dong, Martin F. Schumann, Matti J. Hokkanen, Bo Chang, Alexander Welle, \\ Quan Zhou, Robin H. A. Ras, Zhenliang Xu, Martin Wegener, and Pavel A. Levkin*
}

The ability to create superoleophobic surfaces repellent toward

low-surface-tension liquids is important for various applications, and has been recently demonstrated using re-entrant or doubly re-entrant microtopography. Liquid droplets on such surfaces feature composite liquid-solid-air interfaces, whereas composite liquid-lubricant-air interfaces would have potential for additional repellency. Here, the development of a novel slippery superoleophobic surface with low adhesion is demonstrated via combining doubly re-entrant microtopography with slippery lubricant-infused porous surfaces. This is realized by using 3D direct laser writing to fabricate doubly re-entrant micropillars with dedicated nanostructures on top of each pillar. The top nanostructures stabilize the impregnated slippery lubricant, while the re-entrant geometry of the micropillars prevents lubricant from spreading. The slippery layer reduces the adhesion of liquid to the pillars, as proved using scanning droplet adhesion microscopy (SDAM), while the doubly re-entrant micropillars make the surface superoleophobic. This novel interface combining two extremes, superoleophobicity and slippery lubricant-infused surface, is of importance for designing superoleophobic and superhydrophobic surfaces with advanced liquid repellent, anti-icing, or anti-fouling properties.

and low contact-angle hysteresis (CAH) can find numerous practical applications in self-cleaning, ${ }^{[5]}$ corrosion prevention, ${ }^{[6]}$ drag reduction, ${ }^{[7]}$ and liquid separation. ${ }^{[8]}$ Superhydrophobic surfaces have been developed via a combination of low surface energy of the material and high surface roughness. ${ }^{[9,10]}$ To achieve superoleophobic or superomniphobic surfaces that repel low-surface-tension liquids, re-entrant ${ }^{[11,12]}$ and doubly re-entrant microstructures, ${ }^{[13,14]}$ which can suspend these liquids on top of the microstructures in the Cassie state, were introduced. These surfaces could indeed repel liquids possessing surface tension lower than $15 \mathrm{mN} \mathrm{m}^{-1}$. [13]

When a liquid is suspended in the Cassie state, the liquid-solid contact fraction needs to be low such that the droplet beads up and rolls off the surface. ${ }^{[13]}$ It has been shown previously that reducing liquid-solid contact fraction can effectively reduce the adhesion force (pull-off force) of superhydrophobic surfaces. ${ }^{[15]}$

Controlling surface wettability is important in a wide variety of industrial and research areas ranging from coatings, ${ }^{[1]}$ heat transfer, ${ }^{[2]}$ and liquid manipulation ${ }^{[3]}$ to chemical and biological screening. ${ }^{[4]}$ Liquid super-repellent surfaces inspired by various natural surfaces and possessing both high contact angle (CA)
Reducing liquid-solid contact fraction has been achieved by either increasing the gaps between pillars or reducing the diameter of individual pillars. However, increasing the gaps between pillars leads to a composite interface with low breakthrough pressure, making it susceptible to an irreversible
Z. Dong, Dr. P. A. Levkin

Institute of Toxicology and Genetics

Karlsruhe Institute of Technology

76344 Eggenstein-Leopoldshafen, Germany

E-mail: levkin@kit.edu

Z. Dong, Prof. Z. Xu

State Key Laboratory of Chemical Engineering

East China University of Science and Technology

200237 Shanghai, P. R. China

Dr. M. F. Schumann, Prof. M. Wegener

Institute of Nanotechnology and Institute of Applied Physics

Karlsruhe Institute of Technology

76021 Karlsruhe, Germany

Dr. M. J. Hokkanen, Prof. Q. Zhou

Department of Electrical Engineering and Automation

Aalto University School of Electrical Engineering

02150 Espoo, Finland
Dr. M. J. Hokkanen, Dr. B. Chang, Prof. R. H. A. Ras

Department of Applied Physics

Aalto University School of Science

02150 Espoo, Finland

Dr. B. Chang

College of Mechanical and Electrical Engineering

Shaanxi University of Science and Technology

710021 Xi'An, P. R. China

Dr. A. Welle

Institute of Functional Interfaces and Karlsruhe Nano Micro Facility Karlsruhe Institute of Technology

76344 Eggenstein-Leopoldshafen, Germany

Prof. R. H. A. Ras

Department of Bioproducts and Biosystems

Aalto University School of Chemical Engineering

02150 Espoo, Finland 
transition to the Wenzel state. ${ }^{[16]}$ Reducing pillar diameter also results in a decrease of adhesion force, and has been exemplified in "nanograss" superhydrophobic surfaces with ultralow adhesion force. ${ }^{[17]}$ Nevertheless, this method is difficult to apply for low-surface-tension liquids, which usually require special re-entrant or doubly re-entrant topography.

An alternative strategy to reduce the adhesion force of a droplet is by introducing a defect-free and molecularly smooth liquid-liquid interface instead of the imperfect and chemically heterogeneous solid-liquid interface. ${ }^{[18]}$ This concept has been exemplified by slippery liquid-infused porous surfaces (SLIPS) (or liquid-infused surface, LIS) prepared via infusing a lubricating liquid into a nano/microstructured substrate, ${ }^{19-23]}$ which renders the surface with low sliding angles for various liquids with surface tension ranging from 72.4 down to $17.2 \mathrm{mN} \mathrm{m}^{-1} \cdot{ }^{[19]}$ In addition, due to the dynamic, ultrasmooth nature of a liquid interface, SLIPS have been shown to possess unique features such as anti-biofouling, ${ }^{[24-26]}$ anti-icing, or antifrosting properties. ${ }^{[27]}$ To overcome the gradual loss of infused lubricant in SLIPS, self-lubricating organogels, capable of autonomously supplying functional liquids to the surface, have also been reported. ${ }^{28]}$ However, SLIPS typically show much lower droplet shedding velocities compared to conventional super-repellent surfaces. ${ }^{[29]}$

Here, we aim to combine the properties of the Cassie-state superoleophobic and superhydrophobic surfaces with the slippery, ultrasmooth nature of a liquid-infused surface. Such a surface could be interesting from the fundamental, engineering, and practical prospective. This combination would lead to a Cassie state where liquid droplets rest on a liquid-air (lubricant-air) composite interface, instead of a solid-air surface in common superhydrophobic or superoleophobic surfaces (Figure 1A). Potential applications of such surfaces include superoleophobic and superhydrophobic surfaces with improved liquid repellency, liquid mobility, low adhesion, anti-icing, and anti-fouling properties.

Direct laser writing (DLW) is an additive manufacturing technique based on two-photon polymerization, enabling the making of nearly arbitrary 3D microstructures. ${ }^{[30]}$ Possessing the true freedom for 3D fabrication, as well as the high spatial resolution in sub-micrometer range, DLW has been used for realizing a range of complex structures for applications in photonics, ${ }^{[31]}$ microdevices, ${ }^{[32]}$ and cell research. ${ }^{[33]}$ In this study, we utilize the flexibility of DLW to fabricate doubly re-entrant micropillars with a dedicated nanoroughness on top of the micropillars (Figure 1B). This micro-nano hierarchical pillar structure is similar to the two length scales of topographies, which are important for designing stable superhydrophobic surfaces. ${ }^{[10]}$ The doubly re-entrant topography is crucial to ensure the confinement of a lubricant on top of the micropillars and to achieve superoleophobicity, while the secondary nanoroughness stabilizes the lubricant layer. The wetting properties of the novel slippery superoleophobic surface were investigated using both macroscopic contact angle measurement and a newly developed scanning droplet adhesion microscopy (SDAM), which can measure normal adhesion forces with spatial resolution down to $10 \mu \mathrm{m}$ and three orders of magnitude better force sensitivity than current tensiometers. ${ }^{[17]}$

Combination of SLIPS with superoleophobicity is difficult to achieve by conventional approaches because doubly re-entrant micropillars are commonly fabricated via micromachining with sophisticated equipment and techniques.[13] These lack the freedom to arbitrarily tailor the microstructures for stabilizing the slippery lubricant layer. In addition, to construct a stable lubricant layer on top of the micropillars without contaminating
(A)

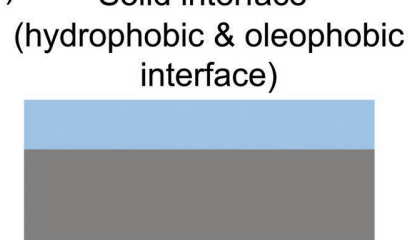

Solid-air interface (Cassie interface)

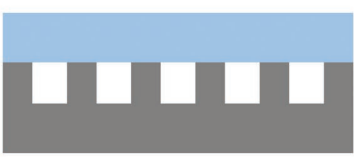

\section{Liquid interface (slippery interface)}

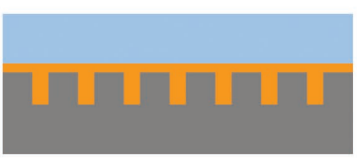

Liquid-air interface (slippery Cassie interface)

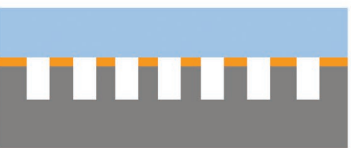

(B)

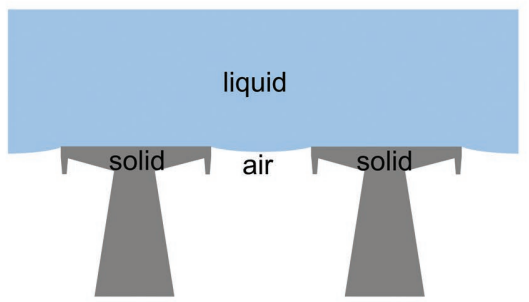

Superoleophobic doubly re-entrant

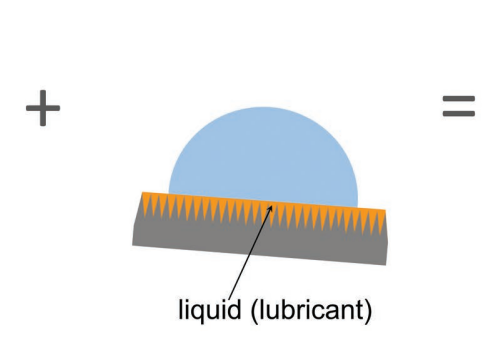

Slippery lubricant-infused porous surface

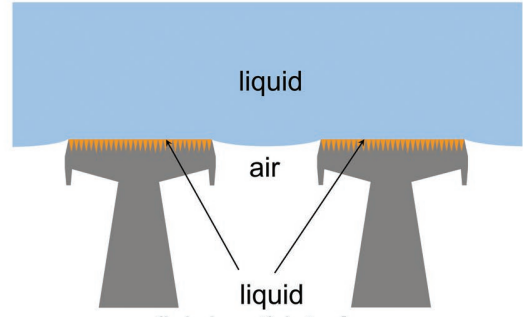

(lubricant) interface

Slippery superoleophobic doubly re-entrant

Figure 1. A) Schemes showing liquid on a hydrophobic \& oleophobic, superhydrophobic \& superoleophobic, slippery and slippery superhydrophobic $\&$ superoleophobic surfaces possessing different contact interfaces. B) Blueprint for a slippery superoleophobic surface formed via combination of a doubly re-entrant structure and a slippery lubricant-infused porous surface. 

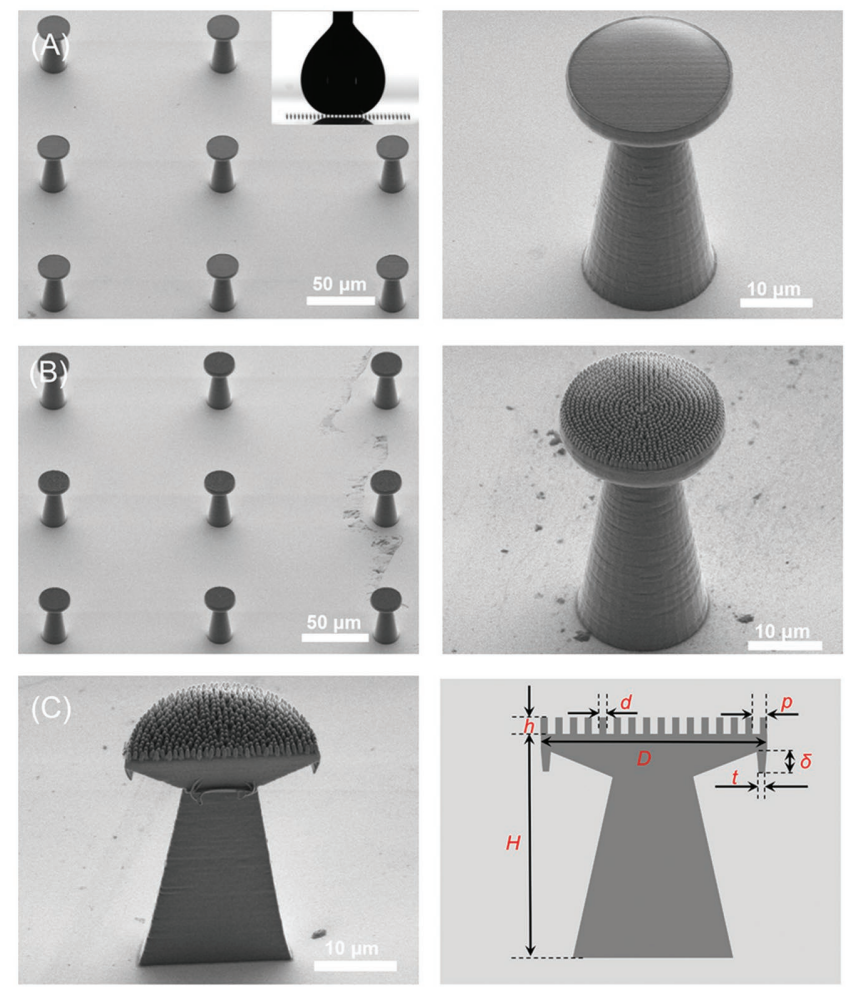

Figure 2. SEM images of doubly re-entrant micropillars with flat and nan opillared (nanorough) tops. A) $45^{\circ}$ view of doubly re-entrant micropillars with flat tops. The inset picture shows a $6 \mu \mathrm{L}$ ethanol drop deposited on a surface composed of the doubly re-entrant micropillars with static contact angle of $158 \pm 5^{\circ}$. B) $45^{\circ}$ view of doubly re-entrant micropillars with nanorough tops. C) Cross-sectional view of a doubly re-entrant micropillar with a nanorough top. Key geometric parameters: $D, P$, and $H$ are the diameter, pitch (center-to-center), and height of the doubly re-entrant micropillars, respectively. $\delta$ and $t$ are the length and thickness of the vertical overhang, respectively. $d, p$, and $h$ are the diameter, pitch (center-to-center), and height of the nanopillars, respectively. $D=25 \mu \mathrm{m}$, $P=100 \mu \mathrm{m}, H=50 \mu \mathrm{m}, \delta=4.0 \mu \mathrm{m}, t=250 \mathrm{~nm}, d=400 \mathrm{~nm}, p=750 \mathrm{~nm}$, $h=1.0 \mu \mathrm{m}$

the interpillar gaps requires a precise manipulation system for the deposition of lubricant oil.

We first utilized DLW to fabricate doubly re-entrant micropillars with flat and nanopillared (nanorough) tops, and the fabricated structures were characterized by scanning electron microscopy (SEM) (Figure 2). The hierarchical micro-nanostructures were fabricated by DLW using a commercial acrylate-based photoresist. The structures were then coated with $50 \mathrm{~nm}$ of $\mathrm{Al}_{2} \mathrm{O}_{3}$ by atomic layer deposition (ALD), followed by fluorination with $1 \mathrm{H}, 1 \mathrm{H}, 2 \mathrm{H}, 2 \mathrm{H}$-perfluorooctyl-trichlorosilane (see the Experimental Section). The successful fluorination of the doubly re-entrant micropillars was proved by the time-of-flight secondary ion mass spectrometry and X-ray photoelectron spectroscopy characterizations (Figures S1 and S2, Supporting Information). A flat $\mathrm{Al}_{2} \mathrm{O}_{3}$ surface fluorinated in the same way showed advancing and receding water contact angles of $124.3 \pm 1.2^{\circ}$ and $82.7 \pm 1.9^{\circ}$, respectively.

To minimize solid-liquid contact fraction $\left(f_{\mathrm{s}}\right)$, the doubly reentrant micropillars with diameter $(D)$ of $25 \mu \mathrm{m}$ are arranged in a square array $\left(3 \times 3 \mathrm{~mm}^{2}\right)$ with a pitch $(P)$ of $100 \mu \mathrm{m}$. The length and thickness of the overhang is $4 \mu \mathrm{m}$ and $250 \mathrm{~nm}$, respectively, resulting in a solid fraction, $f_{\mathrm{s}}$, of $5-8 \%$ depending on the pinning position of the liquid-air interface. The sharp overhang topography of the micropillars can be clearly seen from Figure 2C. Liquid droplets with different surface tension including water $\left(\gamma=72.8 \mathrm{mN} \mathrm{m}^{-1}\right)$, ethanol $\left(\gamma=22.0 \mathrm{mN} \mathrm{m}^{-1}\right)$ and $n$-octane $\left(\gamma=21.1 \mathrm{mN} \mathrm{m}^{-1}\right)$ could bead up and roll off from the micropillar array with sliding angles less than $10^{\circ}$, confirming the superoleophobicity of the doubly re-entrant micropillars (Video S1, Supporting Information). To construct secondary nanoroughness for stabilizing the slippery lubricant layer, nanopillars with diameter $400 \mathrm{~nm}$, pitch $750 \mathrm{~nm}$, and height $1.0 \mu \mathrm{m}$ were fabricated on top of the doubly re-entrant micropillars. Figure 2B,C shows the uniformly distributed nanopillars on top of the micropillars.

In order to create a slippery lubricant layer on top of the doubly re-entrant micropillars without contaminating the gaps between the pillars, we adopted the robotic microdroplet deposition setup shown in Figure 3A. A capillary tip with precise movement control mechanism was dipped into a lubricant oil Krytox 103 and then softly touched the top of the doubly re-entrant micropillars. The whole process was visualized by a high-resolution optical microscope (Figure 3B). From the microscopy images, we can see that a lubricant layer was successfully created on top of the micropillars (Figure S3, Supporting Information). The lubricant coating layer was further confirmed by environmental scanning electron microscopy (ESEM) (Figure 3C). It has also been observed that the lubricant coating layer on the pillar top gradually evaporated in the ESEM chamber due to the low pressure (1.0 Torr).

We first investigated the wetting properties of the slippery lubricant coated micropillars by macroscopic contact angle measurements. Doubly re-entrant micropillar arrays $(D=25 \mu \mathrm{m}, P=100 \mu \mathrm{m})$ with three different top surface, namely, flat and nanorough surfaces without lubricant coating and lubricant-infused nanorough surfaces, were tested using three liquids with different surface tension and polarity including water, ethanol, and $n$-octane. Table 1 shows that the apparent advancing angles $\left(\theta_{\mathrm{a}}^{*}\right)$ of all surfaces remain around $160^{\circ}$, independent of the top surface structure and liquid surface tension. This trend of $\theta_{\mathrm{a}}{ }^{*}$ is consistent with previous reports. For example, Butt and co-workers observed that $\theta_{\mathrm{a}} *$ on superrepellent surface is close to $180^{\circ}$ using confocal microscopy. ${ }^{[34]}$ The underestimation of $\theta_{\mathrm{a}} *$ measured here with goniometer is mainly due to gravitational distortion, which is estimated to cause $\approx 20^{\circ}$ difference for a $5-10 \mu \mathrm{L}$ drop on a completely nonwetting surface. ${ }^{[35]}$ On the other hand, the apparent receding angles $\left(\theta_{\mathrm{r}}^{*}\right)$ is influenced more by the surface and liquid used for the measurements. For water, the lubricant coating leads to an increase of the $\theta_{\mathrm{r}}^{*}$ from $142^{\circ}$ to $148.6^{\circ}$ on nanorough micropillars. However, for ethanol and $n$-octane with low surface tension, the lubricant coating has no obvious influence on the $\theta_{\mathrm{r}} *$. These results are consistent with the $\theta_{\mathrm{r}}^{*}$ saturation effect observed by Dufour et al., who found that when the intrinsic receding angle on a smooth surface $\left(\theta_{\mathrm{r}}\right)$ is higher than the threshold angle $\theta_{\mathrm{t}}$, increasing $\theta_{\mathrm{r}}$ would result in a decrease of $\mathrm{CAH}$ and therefore an increase in $\theta_{\mathrm{r}}{ }^{*}$. On the other hand, when $\theta_{\mathrm{r}}$ is lower than $\theta_{\mathrm{t}}$, CAH remains constant therefore $\theta_{\mathrm{r}}{ }^{*}$ 

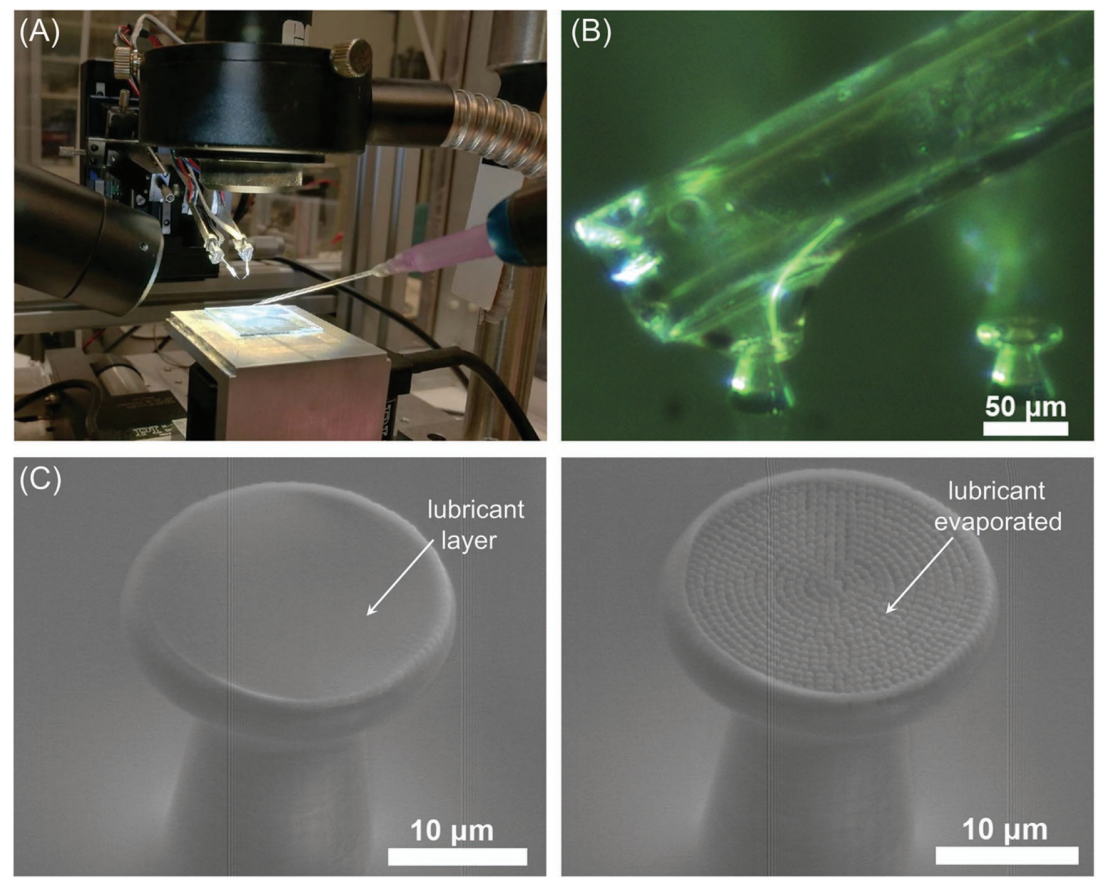

Figure 3. Lubricant oil coating on doubly re-entrant micropillars. A) Experimental setup of the robotic microdroplet deposition device. B) Lubricant oil coating on micropillars using a capillary tip. C) ESEM images of lubricant oil coated nanorough micropillars (pillar diameter $D=25 \mu \mathrm{m}$ ) before (left) and after (right) lubricant evaporation, due to the low environmental pressure (1.0 Torr) in the ESEM chamber.

is not affected by increasing $\theta_{\mathrm{r}}{ }^{[36]}$ It has been proposed that the energy dissipation of moving droplets in the Cassie state involves two terms: the energy corresponding to wetting/dewetting of defects even on a nominally flat surface and the energy stored in the deformation of receding contact line. ${ }^{[36,37]}$ For low-surface-tension liquids, the deformation energy is dominant, and therefore replacing the solid top of the micropillars with lubricant top has little effect on the overall CAH, leading to the same $\theta_{\mathrm{r}}^{*}$. For high-surface-tension liquids like water, the contribution of wetting/dewetting energy is not negligible any more, the lubricant top surface virtually eliminates the surface defects and reduces the wetting/dewetting energy, therefore the overall CAH is reduced and $\theta_{\mathrm{r}}{ }^{*}$ is increased. ${ }^{[36]}$

Unfortunately, contact angle measurements by goniometer are inherently not accurate due to the difficulties in precise positioning of the baseline, construction of tangent lines, and gravitational distortion. This inaccuracy becomes increasingly significant when the contact angles are higher than $150^{\circ} .{ }^{[38]}$ It has been shown that only a $30 \mu \mathrm{m}$ movement of the base line could change the measured apparent contact angle from $160^{\circ}$ to $180^{\circ}{ }^{[39]}$ In addition, as contact angle measurements are based on observing contact line movement on multiple micropillars, it is impossible to detect wetting variations on an individual micropillar. To overcome these problems, we adopted a new scanning droplet adhesion microscopy recently developed by Liimatainen et al. ${ }^{[17]}$ to study the wetting properties of the slippery doubly re-entrant micropillars. Using a vertically mounted force sensor with a liquid droplet probe and a multiaxis sample stage, this technique can measure normal forces point-bypoint with spatial resolution down to $10 \mu \mathrm{m}$ and force sensitivity down to $5 \mathrm{nN}$, providing us an effective way to investigate the influence of the top lubricant layer on the droplet adhesion force of an individual micropillar.

For the droplet adhesion force measurements, $50 \mu \mathrm{m}$ diameter micropillars (Figure S4, Supporting Information) with four different functionalizations of their top surface were used, namely, flat and nanorough top surfaces, with and without lubricant coating. For each type of micropillars, five repetitive measurements were performed on five individual micropillars to verify the accuracy and repeatability. Figure 4A shows a typical force curve for an uncoated micropillar with a flat top, where the snap-in and pull-off forces were obtained as the individual micropillar first made contact with the droplet, and then subsequently detached from it. ${ }^{[17]}$ For micropillars with flat tops, the pulloff force of uncoated micropillars remained constant around $10 \mu \mathrm{N}$ during five consecutive measurements (Figure 4B). After coating the top surface with the lubricant, the pull-off force on the micropillars showed a drastic decrease to around $5 \mu \mathrm{N}$ in the first measurement (Figure 4B and Figure S5, Supporting Information), suggesting that the lubricant layer decreased the adhesive force on the micropillars. However, the pull-off force on lubricant-coated micropillars reached the same order of magnitude as on the uncoated flat micropillars after the first measurement, indicating the very low stability of the lubricant layer on the flat tops. For micropillars with nanorough tops, the uncoated micropillars also showed a high pulloff force of around $10 \mu \mathrm{N}$ (Figure 4C). It is interesting to note that the second level of nanostructures did not result in a difference of pull-off force in dry state between the micropillars with flat tops and nanorough tops. One would expect that the

Table 1. Apparent advancing $\left(\theta_{\mathrm{a}}^{*}\right)$ and receding $\left(\theta_{\mathrm{r}}^{*}\right)$ contact angles of water, ethanol, and $n$-octane on doubly re-entrant micropillars with different top surfaces (pillar diameter $D=25 \mu \mathrm{m}$, pitch $P=100 \mu \mathrm{m}$ ).

\begin{tabular}{|c|c|c|c|c|c|c|c|}
\hline \multirow[t]{2}{*}{ Liquid } & \multirow[t]{2}{*}{ Surface tension $\left[\mathrm{mN} \mathrm{m}^{-1}\right]$} & \multicolumn{2}{|c|}{$\theta^{*}$ on micropillars with flat tops } & \multicolumn{2}{|c|}{$\theta^{*}$ on micropillars with nanorough tops } & \multicolumn{2}{|c|}{$\theta^{*}$ on micropillars with lubricant coating } \\
\hline & & $\theta *{ }_{a}$ & $\theta * *_{r}$ & $\theta *{ }_{a}$ & $\theta *_{\mathrm{r}}$ & $\theta *{ }_{a}$ & $\theta * *_{\mathrm{r}}$ \\
\hline Water & 72.8 & $162.6 \pm 0.9^{\circ}$ & $139.9 \pm 0.7^{\circ}$ & $160.7 \pm 1.3^{\circ}$ & $142.0 \pm 0.8^{\circ}$ & $160.7 \pm 1.2^{\circ}$ & $148.6 \pm 2.2^{\circ}$ \\
\hline Ethanol & 22.0 & $160.5 \pm 0.8^{\circ}$ & $135.6 \pm 1.5^{\circ}$ & $160.2 \pm 0.9^{\circ}$ & $135.2 \pm 0.7^{\circ}$ & $160.0 \pm 1.3^{\circ}$ & $134.3 \pm 0.6^{\circ}$ \\
\hline$n$-Octane & 21.1 & $159.7 \pm 1.2^{\circ}$ & $135.0 \pm 0.8^{\circ}$ & $161.3 \pm 1.1^{\circ}$ & $134.3 \pm 0.9^{\circ}$ & $160.5 \pm 0.8^{\circ}$ & $133.0 \pm 1.7^{\circ}$ \\
\hline
\end{tabular}




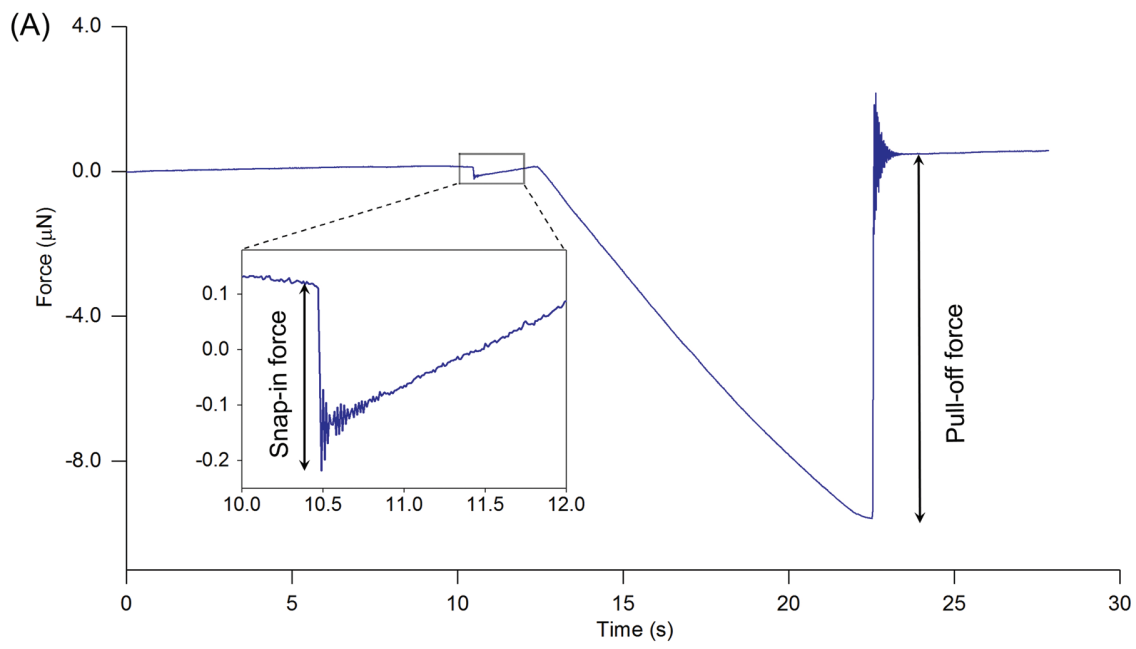

(B)

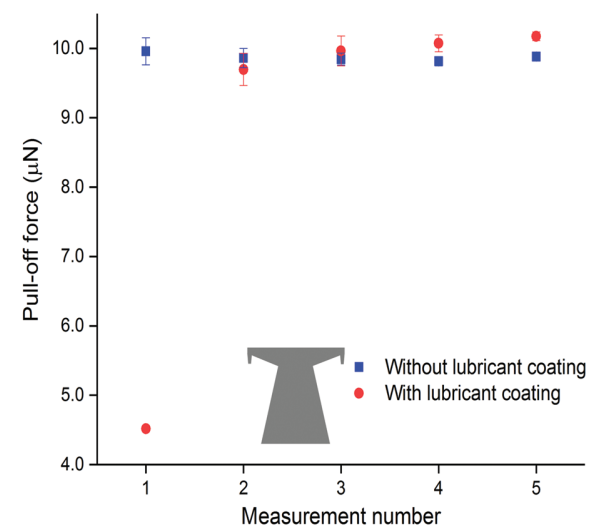

(D)

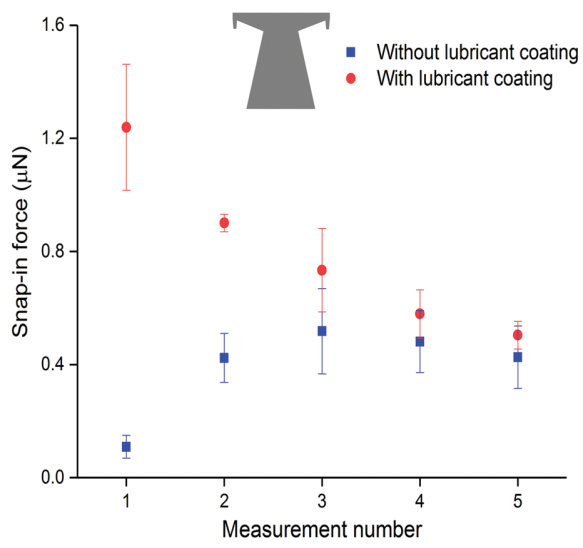

(C)

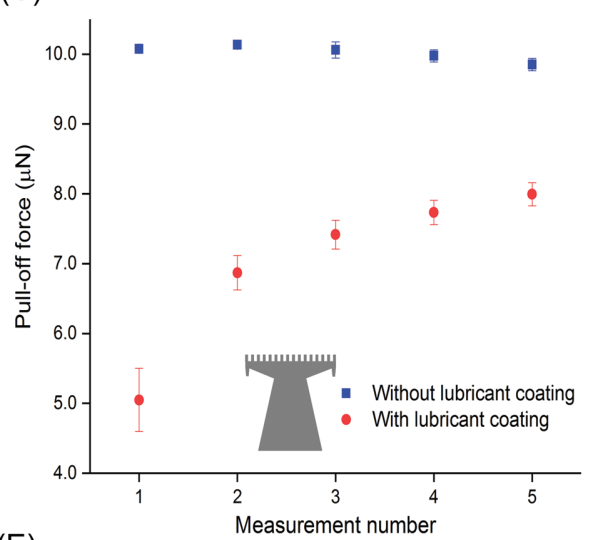

(E)

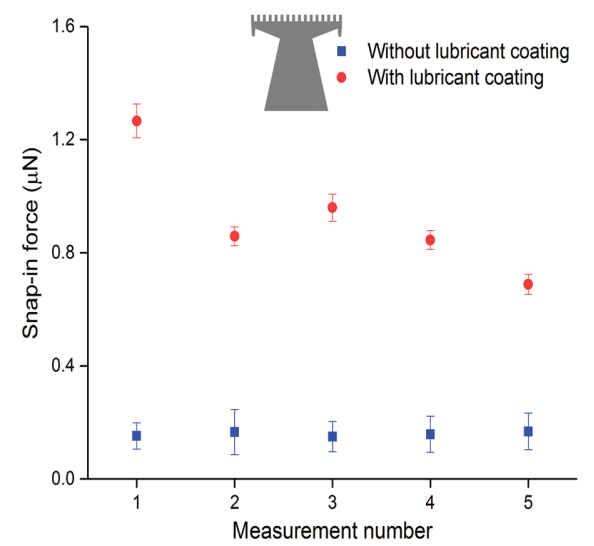

Figure 4. Microscopic droplet adhesion force measurements on individual micropillars. A) A representative force curve for a single $50 \mu \mathrm{m}$ diameter uncoated micropillar with a flat top using a $1.5 \mu \mathrm{L}$ water droplet. B,C) Pull-off forces and D,E) snap-in forces on $50 \mu \mathrm{m}$ diameter micropillars with (red circles) and without (blue squares) lubricant coating. (B) and (D): pillars with flat top surfaces; (C) and (E): pillars with nanorough top surfaces. The error bars represent standard deviations based on 5 independent measurements on five individual micropillars.

adhesion force should either decrease if the nanostructures support a Cassie wetting state or increase if the nanostructures are wetted by the liquid. This could be explained by how the pull-off force is recorded in SDAM measurements. In SDAM measurements, the pull-off force is the maximum tensile force measured by the force sensor during the pull-off step as the droplet separates from the surface. When the separation is clean and water fully detaches from the surface without residue, the pull-off force is the adhesion force between the water droplet and the surface at vertical separation. On the other hand, when the separation is not clean, a residual water droplet remains on the surface following the release, and the force measurement is affected by the cohesion of the liquid. We observed this in the measurements of $50 \mu \mathrm{m}$ diameter 
micropillars. In this case, the recorded high pull-off force $(\approx 10 \mu \mathrm{N})$ is the force needed to break the water droplet, and the magnitude of this force is limited by the cohesive force of the water droplet. ${ }^{[40]}$ Therefore, the measured pull-off force did not show significant differences between flat and nanorough micropillars. Similar to flat micropillars, the lubricant layer again reduced the pull-off force of nanorough micropillars down to $5 \mu \mathrm{N}$, however, this time the pull-off force did not increase to the original $10 \mu \mathrm{N}$ after the first measurement and was slowly approaching $8 \mu \mathrm{N}$ after five consecutive measurements on the same micropillar. The lubricant stability on nanorough pillars was further investigated by 20 consecutive adhesion force measurements and the lubricant-coated pillars showed a steady yet lower pull-off force than uncoated pillars after ten measurements (Figure S6, Supporting Information). The pull-off force on lubricant coated pillars was lower than that on the uncoated pillars even after 20 consecutive measurements, indicating that the secondary nanoroughness indeed stabilizes the lubricant layer. On the other hand, the lubricant layer increased the snap-in force on the micropillars (Figure 4D,E), which might be attributed to the decreased water advancing contact angle obtained on flat nonstructured slippery lubricant-infused surfaces (Table S1, Supporting Information) or to the lubricant cloaking effect. For lubricant-coated flat micropillars, the snap-in force decreased to the same order of magnitude as on uncoated flat micropillars after five measurements, while for the lubricant-coated nanorough micropillars, the snap-in force showed a steadier decrease (Figure 4). This trend is similar to that of pull-off force, confirming that the secondary nanoroughness stabilizes the lubricant layer on top of the micropillars.

We further investigated the snap-in and pull-off forces of $n$-octane on $50 \mu \mathrm{m}$ diameter doubly re-entrant micropillars with different top surfaces. The slippery lubricant layer increased the snap-in forces of the micropillars, while the pull-off forces remained almost unchanged (Figure S7, Supporting Information). This could be explained by the incomplete dewetting of the pillars due to rupture of the $n$-octane droplets during the droplet detachment (Video S2, Supporting Information). As discussed previously, the magnitude of the recorded pull-off force in this case is related to cohesive force of the $n$-octane droplet, and the contact area when the droplet breaks, therefore all micropillars displayed almost same values of pull-off force regardless of their varied top functionalities. Different from the SDAM measurements with water, the lubricant layer was more stable during $n$-octane measurements, as both the pull-off and snap-in forces on lubricant-coated pillars remained steady during the five consecutive measurements (Figure S7, Supporting Information). In SDAM measurements, the squeezing out of the lubricant layer underneath the probing liquid droplet might be resulted from the Laplace pressure inside the droplet, which is proportional to the surface tension of the probing liquid. ${ }^{[18]}$ As n-octane $\left(\gamma=21.1 \mathrm{mN} \mathrm{m}^{-1}\right)$ possesses much lower surface tension than water $\left(\gamma=72.8 \mathrm{mN} \mathrm{m}^{-1}\right)$, the driving pressure squeezing out the lubricant layer was much smaller during $n$-octane measurements, which might be the reason for higher stability of the lubricant layer observed. We also tested the snap-in and pulloff forces of water and $n$-octane on $25 \mu \mathrm{m}$ diameter doubly re-entrant micropillars with different top surfaces (Figures S8 and S9, Supporting Information). In general, both the pull-off forces and snap-in forces showed much lower values on the $25 \mu \mathrm{m}$ diameter micropillars, indicating that decreasing pillar diameter resulted in a decrease of the adhesion force. Consistent with the test results on $50 \mu \mathrm{m}$ diameter micropillars, the slippery lubricant layer also decreased the pull-off force of water on $25 \mu \mathrm{m}$ diameter nanorough micropillars from 5.0 to $4.0 \approx 4.5 \mu \mathrm{N}$ during the five consecutive measurements (Figure S8, Supporting Information).

In conclusion, we have used 3D DLW for rapid prototyping and systematic investigation of arrays of hierarchical doubly re-entrant micropillars with or without nanopillars on their top surfaces. By infusing a lubricant into the nanopillared surface on top of the doubly re-entrant micropillars, we could combine the slippery low adhesion effect of SLIPS with the superoleophobicity of doubly re-entrant microstructures, leading to a new type of Cassie state with a liquid droplet located on the lubricant-air composite interface, avoiding direct contact between droplet and solid interface. The doubly re-entrant nature of the micropillars is important not only to create a superoleophobic surface, but also to keep the lowsurface-tension lubricant on top of the micropillars without spreading into the interpillar gaps. It is interesting to note that the produced slippery superoleophobic surface did not result in a drastic reduction of the contact angle hysteresis (especially for the organic solvents), as could be expected from the "slippery" nature of liquid-infused interfaces, which usually show very low contact angle hysteresis. ${ }^{[19]}$ However, this can be explained by generally low contact angles (both receding and advancing) obtained on flat slippery lubricant-infused surfaces, for example, the advancing angle and receding angle of $n$-octane on a regular SLIPS is $47.2 \pm 0.5^{\circ}$ and $46.1 \pm 0.7^{\circ}$, respectively (Table $\mathrm{S} 1$, Supporting Information). Nevertheless, the scanning droplet adhesion microscopy provided us a new way to investigate wetting variations on individual micropillars that had previously not been possible. The results revealed that the slippery layer significantly reduced the adhesive force of water droplets on individual micropillars proving the existence of the lubricant-air composite Cassie state on this surface. In addition, the adhesion force measurements confirmed the need for the nanoroughness in order to stabilize the lubricant layer on the tops of the doubly re-entrant micropillars. We envision that this novel type of slippery superoleophobic surface could be interesting for both fundamental research and practical applications including superoleophobic surfaces with extreme liquid repellency, controlled droplet mobility, anti-icing, anti-fouling, anti-adhesive, or other properties attributed to its lubricant-air hybrid interface. Clearly, in its present form, the DLW is not suitable for the mass fabrication of large footprints. However, in the future, by using arrays of many laser foci, this approach could be made scalable. ${ }^{[41]}$ 


\section{Acknowledgements}

This research was supported by the ERC Starting Grant (337077-DropCellArray), the Helmholtz Association's Initiative and Networking Fund (VH-NG-621), the Helmholtz program Science and Technology of Nanosystems (STN), the Karlsruhe School of Optics \& Photonics (KSOP), the European Research Council ERC-2016-CoC (725513-SuperRepel), Academy of Finland (Centres of Excellence Programme (2014-2019), and the AScl/ELEC Thematic Research Programme. The authors are grateful to Dr. Tawheed Mohamed (KIT) for his help with environmental scanning electron microscopy. Z.D. thanks the China Scholarship Council for a joint Ph.D. scholarship. The authors are grateful to Dr. Alexei Nefedov (IFG, KIT) for helping with the XPS measurements.

\section{Conflict of Interest}

The authors declare no conflict of interest.

\section{Keywords}

direct laser writing, doubly re-entrant, low adhesion force, slippery surfaces, superoleophobicity

[1] X. Deng, L. Mammen, H.-J. Butt, D. Vollmer, Science 2012, 335, 67.

[2] A. R. Betz, J. Jenkins, C.-J. C. Kim, D. Attinger, Int. J. Heat Mass Transfer 2013, 57, 733.

[3] X. Hou, Y. Hu, A. Grinthal, M. Khan, J. Aizenberg, Nature 2015, 519, 70.

[4] W. Feng, E. Ueda, A. L. Pavel, Adv. Mater. 2018, 30, 1706111.

[5] R. Blossey, Nat. Mater. 2003, 2, 301.

[6] S. Pan, A. K. Kota, J. M. Mabry, A. Tuteja, J. Am. Chem. Soc. 2013, 135, 578.

[7] C. Lee, C.-J. Kim, Phys. Rev. Lett. 2011, 106, 014502.

[8] Y. Si, Q. Fu, X. Wang, J. Zhu, J. Yu, G. Sun, B. Ding, ACS Nano 2015, 9, 3791.

[9] X.-M. Li, D. Reinhoudt, M. Crego-Calama, Chem. Soc. Rev. 2007, 36, 1350.

[10] L. Gao, T. J. McCarthy, Langmuir 2006, 22, 2966.

[11] A. Tuteja, W. Choi, M. Ma, J. M. Mabry, S. A. Mazzella, G. C. Rutledge, G. H. McKinley, R. E. Cohen, Science 2007, 318, 1618.

[12] A. Grigoryev, I. Tokarev, K. G. Kornev, I. Luzinov, S. Minko, J. Am. Chem. Soc. 2012, 134, 12916.

[13] T. L. Liu, C.-J. C. Kim, Science 2014, 346, 1096.

[14] J. Choi, W. Jo, S. Y. Lee, Y. S. Jung, S.-H. Kim, H.-T. Kim, ACS Nano 2017, 11, 7821.
[15] K. Koch, B. Bhushan, Y. C. Jung, W. Barthlott, Soft Matter 2009, 5, 1386.

[16] A. Tuteja, W. Choi, J. M. Mabry, G. H. McKinley, R. E. Cohen, Proc. Natl. Acad. Sci. USA 2008, 105, 18200.

[17] V. Liimatainen, M. Vuckovac, V. Jokinen, V. Sariola, M. J. Hokkanen, Q. Zhou, R. H. A. Ras, Nat. Commun. 2017, 8, 1798.

[18] D. Daniel, J. V. I. Timonen, R. Li, S. J. Velling, J. Aizenberg, Nat. Phys. 2017, 13, 1020.

[19] T.-S. Wong, S. H. Kang, S. K. Y. Tang, E. J. Smythe, B. D. Hatton, A. Grinthal, J. Aizenberg, Nature 2011, 477, 443.

[20] Q. David, Rep. Prog. Phys. 2005, 68, 2495.

[21] A. Lafuma, D. Quéré, Eur. Phys. Lett. 2011, 96, 56001.

[22] B. R. Solomon, S. B. Subramanyam, T. A. Farnham, K. S. Khalil, S. Anand, K. K. Varanasi, Non-Wettable Surfaces: Theory, Preparation and Applications, Royal Society of Chemistry, London, UK 2017, p. 285.

[23] J. Smith, R. Dhiman, K. Varanasi, presented at 64th Annual Meeting of the APS Division of Fluid Dynamics, Baltimore, MD, USA, November 2011.

[24] D. C. Leslie, A. Waterhouse, J. B. Berthet, T. M. Valentin, A. L. Watters, A. Jain, P. Kim, B. D. Hatton, A. Nedder, K. Donovan, E. H. Super, C. Howell, C. P. Johnson, T. L. Vu, D. E. Bolgen, S. Rifai, A. R. Hansen, M. Aizenberg, M. Super, J. Aizenberg, D. E. Ingber, Nat. Biotechnol. 2014, 32, 1134.

[25] S. Sunny, G. Cheng, D. Daniel, P. Lo, S. Ochoa, C. Howell, N. Vogel, A. Majid, J. Aizenberg, Proc. Natl. Acad. Sci. USA 2016, 113, 11676.

[26] S. Amini, S. Kolle, L. Petrone, O. Ahanotu, S. Sunny, C. N. Sutanto, S. Hoon, L. Cohen, J. C. Weaver, J. Aizenberg, N. Vogel, A. Miserez, Science 2017, 357, 668

[27] P. Kim, T.-S. Wong, J. Alvarenga, M. J. Kreder, W. E. Adorno-Martinez, J. Aizenberg, ACS Nano 2012, 6, 6569.

[28] C. Urata, G. J. Dunderdale, M. W. England, A. Hozumi, J. Mater. Chem. A 2015, 3, 12626.

[29] M. Cao, D. Guo, C. Yu, K. Li, M. Liu, L. Jiang, ACS Appl. Mater. Interfaces 2016, 8, 3615.

[30] J. Fischer, M. Wegener, Laser Photonics Rev. 2013, 7, 22.

[31] T. Gissibl, S. Thiele, A. Herkommer, H. Giessen, Nat. Photonics 2016, 10, 554

[32] S. Tottori, L. Zhang, F. Qiu, K. K. Krawczyk, A. Franco-Obregón, B. J. Nelson, Adv. Mater. 2012, 24, 811.

[33] B. Richter, V. Hahn, S. Bertels, T. K. Claus, M. Wegener, G. Delaittre, C. Barner-Kowollik, M. Bastmeyer, Adv. Mater. 2017, 29, 1604342

[34] F. Schellenberger, N. Encinas, D. Vollmer, H.-J. Butt, Phys. Rev. Lett. 2016, 116, 096101.

[35] C. W. Extrand, S. I. Moon, Langmuir 2010, 26, 17090.

[36] R. Dufour, M. Harnois, V. Thomy, R. Boukherroub, V. Senez, Soft Matter 2011, 7, 9380.

[37] M. Nosonovsky, B. Bhushan, Nano Lett. 2007, 7, 2633.

[38] S. Srinivasan, G. H. McKinley, R. E. Cohen, Langmuir 2011, 27, 13582.

[39] C. W. Extrand, S. I. Moon, Langmuir 2014, 30, 8791.

[40] B. Samuel, H. Zhao, K.-Y. Law, J. Phys. Chem. C 2011, 115, 14852.

[41] C. Barner-Kowollik, M. Bastmeyer, E. Blasco, G. Delaittre, P. Müller, B. Richter, M. Wegener, Angew. Chem., Int. Ed. 2017, 56, 15828. 
Karlsruher Institut für Technologie

Repository KITopen

Dies ist ein Postprint/begutachtetes Manuskript.

Empfohlene Zitierung:

Dong, Z.; Schumann, M. F.; Hokkanen, M. J.; Chang, B.; Welle, A.; Zhou, Q.; Ras, R. H. A.; Xu, Z.; Wegener, M.; Levkin, P. A.

Superoleophobic Slippery Lubricant-Infused Surfaces: Combining Two Extremes in the Same Surface.

2018. Advanced materials, 30.

doi: $\underline{10.5445 / / R / 1000087513}$

Zitierung der Originalveröffentlichung:

Dong, Z.; Schumann, M. F.; Hokkanen, M. J.; Chang, B.; Welle, A.; Zhou, Q.; Ras, R. H. A.; Xu, Z.; Wegener, M.; Levkin, P. A.

Superoleophobic Slippery Lubricant-Infused Surfaces: Combining Two Extremes in the Same Surface.

2018. Advanced materials, 30 (45), 1803890.

doi:10.1002/adma.201803890 